\title{
Critical Study and Analysis of Musculoskeletal Disorders among Practicing Dentists
}

\author{
Judith Earnesty D J \\ Student, Department of IEM \\ BMS College of Engineering \\ Bangalore, India
}

\author{
Roopa V \\ Student, Department of IEM \\ BMS College of Engineering \\ Bangalore, India
}

Prithvi N

Student, Department of IEM

BMS College of Engineering

Bangalore, India

\author{
Dr. V N Shailaja \\ Associate Professor, Department of IEM \\ BMS College of Engineering \\ Bangalore, India
}

\author{
Dr. B Ravishankar \\ Professor, Department of IEM \\ BMS College of Engineering \\ Bangalore, India
}

\begin{abstract}
In this study, the work situations of dental practitioners and the factors contributing to workload are analyzed based on ergonomics. Problem solving methodology was incorporated. The study aims at identifying the major musculoskeletal disorders among dentists and determining the causes for their prevalence. Dental students and practitioners were surveyed and interviewed for collecting data. Observations and simple statistics were used to understand the data. RULA method was used for assessment of the body posture and movements of dentists at work. The results showed that $\mathbf{7 6}$ percent of them suffer from MSDs and more than 85 percent of them do not take any measures to resolve them. Hand and wrist, lower back and upper back were found to be the most affected regions respectively.
\end{abstract}

Keywords-Dentistry; Ergonomics; Musculoskeletal Disorder (MSD); Occupational safety; Rapid Upper Limb Assessment (RULA)

\section{INTRODUCTION}

Dental profession involves complex processes that deal with the learning and treatment of problems in the oral cavity. Dentistry is not immune from MSDs which are injuries or pain in the musculoskeletal system of humans, namely the joints, ligaments, muscles, nerves, tendons, and structures that support limbs, neck and back. Risk factors for work related MSDs related to dentistry include stress, reduced flexibility, inappropriate positioning, infrequent breaks, repetitive movement, weak postural muscles, extended awkward postures and improper setting of equipment. Proper ergonomic design is necessary to avoid repetitive strain injuries (RSJ), which can develop over time and can lead to long term disability.

\section{RELATED WORK}

Literature review laid out a brief idea about the major problems that are encountered by the dentists and lack of attention given towards ergonomic issues. Musculoskeletal disorders (MSD) are of the highest concern. NIOSH has carried out researches on occupational injury by gathering injury data, stakeholder key, faculty capacity and using tools such as RULA and Nordic questionnaire [1]. Studies are carried out at construction sites to assess ergonomic issues and appropriate statistical models were used to arrive at significant conclusions [2]. A study on dentists includes their sitting position, ergonomic guidelines related to the chair, workstation design, arm supports, choice of instruments, load control, work breaks, stamina escalation, magnification systems and so on [3]. It was found that the lower arm was affected the most and requires support [4]. RULA has identified that the use of proper type of seating may reduce the development of work-related MSDs and help in maintaining suitable working posture [5].

\section{METHODOLOGY ADOPTED}

A five step problem solving methodology was used to analyze and identify the major risk factors as shown in Fig. 1 . The first step started with collection of raw data through various means such as journals, surveys and interviews. The raw data obtained were carefully analyzed to draw conclusions regarding the existence of MSDs among dentists. Further, a decision matrix was drawn to identify the major region affected from MSD. Finally, the major risk factors were identified. 


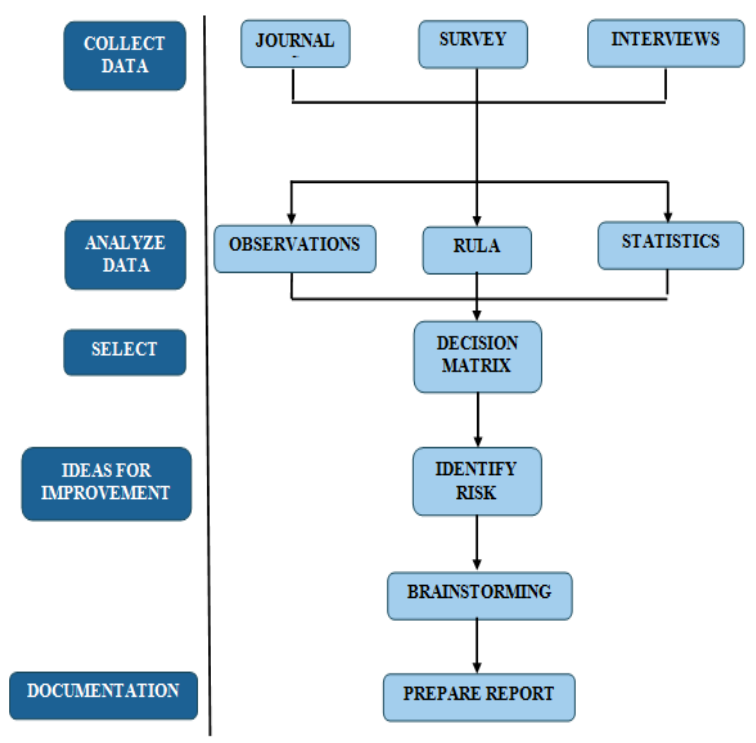

Fig. 1. Methodology

\section{DATA COLLECTION}

Data were collected through journals, surveys and interviews.

\section{A. Journals}

An exhaustive literature survey was carried out to know the research works carried out on occupational safety and health among dental practitioners. Around thirty and odd journals were referred, through which the methods used to carry out the study, procedure followed, major risk factors of consideration and various methods of analyzing were learnt. These were incorporated in the study. The results from literatures were also used as a source of data for the analysis.

\section{B. Surveys}

A survey was carried out among dental practitioners to know the musculoskeletal disorders experienced by them and its effects. The questionnaire contained 15 questions. For measuring the various factors a five-point Likert scale was chosen.

Around 52 responses from people working in various departments of dentistry were received from the survey. It was clearly known that almost all dental practitioners suffer from musculoskeletal pain and discomfort. The survey questionnaire was tested for reliability through Cronbach's alpha test. The test value was found to be 0.79 which suggests that the survey is acceptable. The results from the survey helped to identify the issues which are significant and to address them immediately, rather than waste time on areas of little or no concern.

\section{Interviews}

The dentists were interviewed in their place of work to gather data about incidences of MSDs among them and other factors related to it. These gave an insight of the practical work scenario. Their work was observed and the positions and postures while treating the patients were noted. 10 dentists were consulted and interviewed for a time span of 15-20 minutes.

\section{DATA ANALYSIS}

Data analysis involves inspecting the data, cleansing the information, transforming the data and modelling the data to obtain useful informing conclusions and support decision making.

The data obtained were placed in the form of rows and columns in the excel spreadsheet for further analysis.

Fig. 2, Fig. 3 and Fig. 4 represent the graphs obtained from survey and analysis.

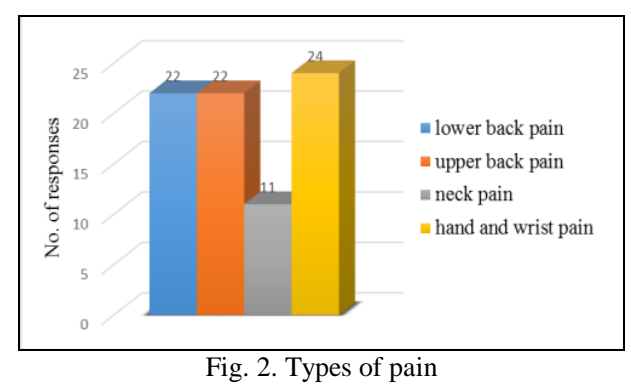

The graphs obtained from survey results clearly indicate the
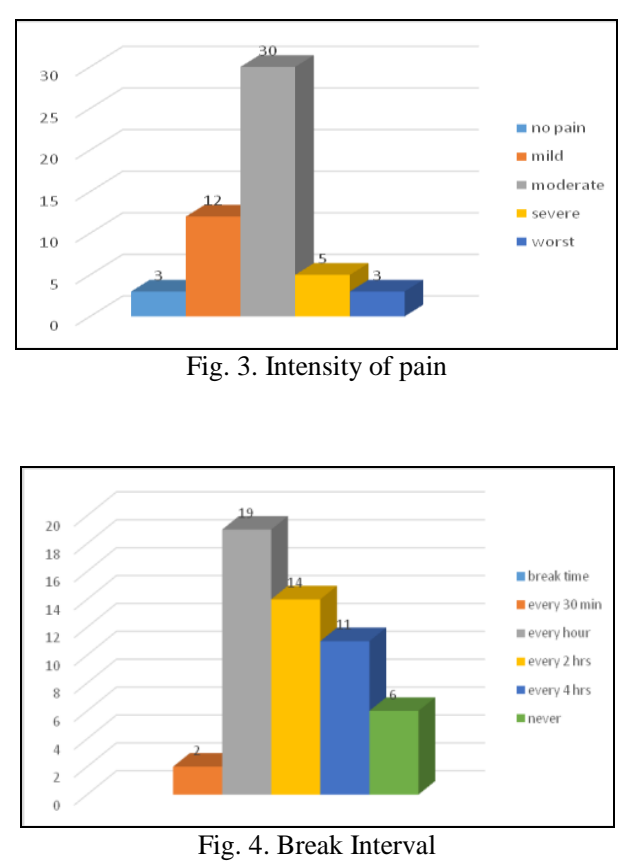

prevalence of pain in different regions of the body and their severity. It was also inferred that they work continuously for long hours without taking sufficient breaks, which is one of the main causes for their existing pain.

\section{ANALYSIS}

\section{A. Ishikawa Diagram}

To identify the root cause for musculoskeletal disorders in dentists, Ishikawa diagram is constructed as in Fig. 5. 


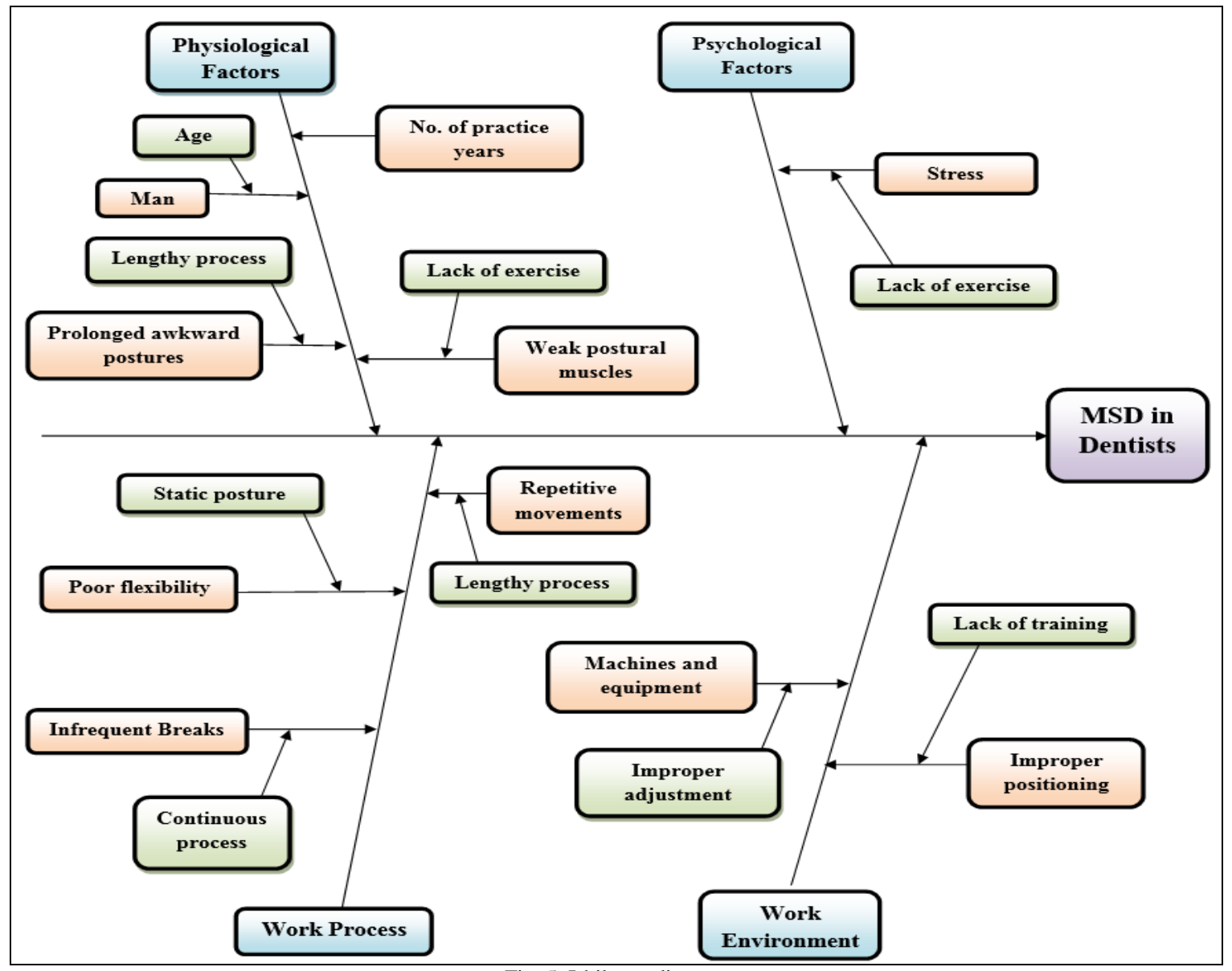

Fig. 5. Ishikawa diagram

The factors were divided into four major groups: physiological, psychological, work process and work environment. The study is carried out based upon these factors.

\section{B. Rapid Upper Limb Assessment (RULA)}

RULA is one of the most widely used tools to analyze the musculoskeletal disorders. Rapid Upper Limb Assessment (RULA) is an ergonomic assessment tool that is used to assess the body postures and disorders related to those postures in a systematic way. It is a survey method of identifying the different postural disorders experienced by the workers. Table I shows the level of MSD Risk based on the score. RULA focuses on the upper limb part of the body. Regions of body considered here are arm, wrist, neck, trunk and legs.

\section{TABLE I. RULA SCORE}

\begin{tabular}{|l|l|}
\hline RULA Score & \multicolumn{1}{c|}{ Level of MSD Risk } \\
\hline $1-2$ & Negligible risk, no action required \\
\hline $3-4$ & Low risk, change may be needed \\
\hline $5-6$ & Medium risk, further investigation, change soon \\
\hline $6+$ & Very high risk, implement change now \\
\hline
\end{tabular}

Selection of the posture for RULA should be based on:

- The most difficult work tasks and posture (based on worker interview and initial observation)

- The posture retained for a longest period of time
- The posture where the maximum force load occurs.

RULA assessment was carried out based on postures adopted, so different postures were evaluated. The postures were selected based on the selection criteria. RULA was carried out on 20 different dental practitioners of both the genders from various departments.

The mean upper arm posture scores were $2.85 \pm 1.66$, while the average lower arms posture scores were $2.05 \pm 0.83$. The average wrist posture scores were $2.50 \pm 0.95$, while the average wrist twist posture scores were $1.55 \pm 0.51$. The mean neck, trunk and legs posture scores were $3.15 \pm 1.23,3.10 \pm 1.41$, $1.55 \pm 0.51$ respectively. The final RULA score ranged from 4 $(\mathrm{n}=5,25 \%)$ to $7(\mathrm{n}=6,30 \%)$ with an average of $5.75 \pm 1.16$.

The RULA tool indicates conveys that there is MSD risk involved in the adopted posture. Out of 20 readings obtained, 5 showed low risk, 9 showed medium risk and 6 assessments showed high level of risk all of which require further investigation and change at the earliest.

\section{Identifying Risk Factors}

Various departments involved in dentistry were selected, baseline data was used to infer risk caused by MSD such as survey questionnaire and expert interviews, and related tasks were identified for further study. Work tasks were taken based 
on the data gathered from each department. Results obtained from this were used as a confirmation of noteworthy potential for MSD risk factor exposure. From the results, it was seen that the explanation of an event and the methods of tracing them varied for every department.

The obtained tasks were incorporated into a table adopted by the NIOSH team. The table was drawn between major risk factors identified and the region of pain. It was found that the dentists suffered slowly and continuously from musculoskeletal discomfort from all the identified risk factors. The risk seemed higher than usual, and a change in their work habits is to be definitely implemented.

\section{Decision Matrix}

A decision matrix was adopted from NIOSH as shown in Table II, to identify the region of body that is most affected by rating the severity of pain determined from each of the sources. The severity of pain was measured using a five point scale starting from 1 for no pain to 5 for the worst case for neck, hand and wrist, upper back and lower back.

The severity of pain in these regions was rated based on journal data, survey data, interviews and RULA test.

The final score was calculated by taking the sum of the scores from each source. For example, for the neck region, the scores would be 2 for mild and 3 for moderate. The final score would be $2+3+3+2=10$. The final scores from the matrix indicated that the pain in hand and wrist was the highest with a score of 16 , followed by lower back and upper back pain with scores of 15 and 13 respectively. The neck region can be neglected as it has a low score of 10.

\section{TABLE II. DECISION MATRIX}

\begin{tabular}{|c|c|c|c|c|c|c|}
\hline \multirow{2}{*}{$\begin{array}{c}\text { Area } \\
\text { of } \\
\text { pain }\end{array}$} & \multirow{2}{*}{$\begin{array}{c}\text { No. of } \\
\text { Incidents }\end{array}$} & \multicolumn{4}{|c|}{ Severity of Pain } & \multirow{2}{*}{ Final } \\
\cline { 3 - 6 } & data & $\begin{array}{c}\text { Survey } \\
\text { data }\end{array}$ & $\begin{array}{c}\text { Intervie } \\
\boldsymbol{w}\end{array}$ & RULA & Score \\
\hline Neck & 32 & Mild & Medium & Medium & Mild & 10 \\
\hline $\begin{array}{c}\text { Hand } \\
\text { and } \\
\text { wrist }\end{array}$ & 21 & Worst & Severe & Medium & Severe & 16 \\
\hline $\begin{array}{c}\text { Uppe } \\
\text { r back }\end{array}$ & 32 & Medium & Medium & Severe & Medium & 13 \\
\hline $\begin{array}{c}\text { Lowe } \\
\text { r back }\end{array}$ & 32 & Severe & Severe & Severe & Medium & 15 \\
\hline
\end{tabular}

\section{RESULT}

The study suggests a high prevalence of about $76 \%$ of dentists suffering from musculoskeletal pain, among which $75 \%$ took no remedial measures. The result of this study also identifies certain major risk factors for the development of the pain. Some of them are prolonged awkward postures, infrequent breaks, lack of exercise, improper positioning and adjustment of equipment. The results from the analysis also highlighted hand and wrist, upper and lower back as the major body regions affected by MSD.

\section{CONCLUSION}

This study was carried on practicing dentists to identify the ergonomic issues faced by them. Many of them were prone to pain which they considered to be an inevitable part of the work of their profession. They do not specifically know what has caused the pain. Hence endure the pain and continue to work without taking any measures to reduce it. Analysis showed that musculoskeletal pain is multifactorial in origin.

Hence it is advised that the dentists carefully consider the acceptance of suitable strategies to help minimize the impact of MSD and ergonomics be introduced as a part of the curriculum of the dental students right from their commencement in dentistry.

\section{REFERENCES}

[1] William J. Wiehagen and Fred C. Turin, "Ergonomic assessment of musculoskeletal risk factors at four mine sites: underground coal, surface copper, surface phosphate, and underground limestone, national institute for occupational safety and health," Publication No. 200W159.

[2] Ravishankar, B., Shailaja V. N., and Bhandarkar, B. M., "A critical study, on human factors leading to stresses, in women at a typical garment industry," 2017 [APIEMS Conf., Indonesia, June 2017].

[3] Shipra Gupta, "Ergonomic applications to dental practice," Indian Journal of Dental Research, 2011, 22(6)

[4] Sareh Moosavi, Rahul Desai, Shaygan Hallaj, K. K. Sundaram, Vivek S Hegde, "Ergonomic analysis to study the intensity of msds among practicing indian dentists," Procedia Manufacturing 3, 2015, pp. $5419-$ 5426.

[5] A. Gandavadi, J. R. E. Ramsay and F. J. T. Burke, "Assessment of dental student posture in two seating conditions using RULA methodology-a pilot study," British Dental Journal, vol. 203, no. 10, November 2007.

[6] Shailaja V. N., \& Narkhed, R. B., "Modeling a stress index with multivariate parameters under a typical engineering environment," IJRBAT, issue (VI), vol. 1, pp. 63-68, January 2018. 